\title{
Epidemiological study of pathogens collected from blood for a period of a year (2008-2009)
}

\author{
Eugenio A. Debbia', Elisabetta Maioli' ${ }^{1}$, Maddalena Perotti ${ }^{1}$, Roberto Bandettini' ${ }^{2}$, Rosalba Bona3, \\ Luigi Carlo Bottaro4, Orietta Illiberi", Roberto Capuzzo5, Bruno Massone, Mariella Dono, \\ Pier Andrea Dusi', Rita Revello7, Maria Gabriella Mazzarello;, Gian Luigi Devoto', Silvia Reali, \\ Monica Marangoni ${ }^{9}$, Giorgio Annovazzi ${ }^{9}$, Teresa Borreanaz ${ }^{9}$, Luisa Santoriello ${ }^{10}$, \\ Domizio Serra ${ }^{11}$, Marco Mori" ${ }^{12}$, David Usiglio" ${ }^{12}$, Anna Marchese', \\ Gruppo di Studio Interregionale sulle Resistenze agli Antibiotici, Sezione di Microbiologia \\ - DISCMIT, Università di Genova
}

Laboratorio di M icrobiologia Clinica e Sperimentale, Sezione di M icrobiologia, Università di Genova'; Istituto Giannina Gaslini, Genova2; ASL2 Ospedale San Paolo, Savona³; ASL3 Ospedale San Carlo, Genova-Voltri4; Ospedale "Villa Scassi", Genova-Sampierdarena ${ }^{5}$; ASL 5 Spezzina, Ospedale Civile S. Andrea, La Spezia ${ }^{6}$;ASL 1 Imperiese, Ospedale di Sanremo, (Imperia); ; ASL 22 Ovada (Alessandria) ${ }^{8}$; ASL4 Chiavarese, Genova9; Ospedale Santa Corona Hospital, Pietra Ligure (Savona) ${ }^{10}$; Ospedale Evangelico Internazionale, Genova ${ }^{11}$; Ente Ospedaliero "Galliera", Genova ${ }^{12}$

Key Words: antibiotic resistance, blo od, N orthern Italy, MRSA, ceftazidime-resistance

Studio epidemiologico di patogeni isolati da emocolture per il periodo di un anno (2008-2009)

\section{SUMMARY}

Objectives. A n epidemio logical study, addressed to identify the pathogens isolated from blood, and their antibiotic susceptibility patterns, was conducted.

Methods. 12 laboratories, homogeneously distributed in a N orthern area of Italy, were required to collected all consecutive non-duplicated strains isolated from blood during February 2008 to February 2009 and sent them to the reference laboratory.

Results. A total of 1092 microorganisms were collected, including 653 gram-positive, 385 gram-negative and 54 fungi. Escherichia coli 234 , Staphylococcus epidermidis 205 , S. aureus 142 , S. hominis 87 , Enterococcus faecalis 47 , S. haemolyticus 33 , Klebsiella pneumoniae 33, Pseudomonas aeruginosa 32, Candida albicans 28, Enterobacter cloacae 21 were the prevalent microrganisms found. Samples were collected mainly from medicine (255 strains), intensive care units (154), surgery (99), infectious diseases (93), paediatrics (62) and nephrology (62). Antibiotic resistance (in \%) in staphylococci was 65.7 (methicillin), 33.5 (gentamicin), 61.8 (azithromycin), 59.6 (er ythromycin), 45.2 (ciprofloxacin) 14.8 (chloramphenicol), 2.0 (teicoplanin), and 24.1 (trimethoprim-sulfamethoxazole) no vancomycin-resistant strain was found. Enterococci showed resistance to vancomycin (10.8), ampicillin (34.4), gentamycin (42.9), ciprofloxacin (42.2) teicoplanin (7.6), erythromycin (54.7) and chloramphenicol (17.5). Enterobacteriaceae exhibited resistance to ciprofloxacin(27.0), ampicillin (74.1), ceftazidime (15.8), cefoxitin (14.7), cefepime (13.3), ceftriaxone (15.0), both imipenem and amikacin (0.95), piperacillin-tazobactam (5.1) and trimethoprim-sulfamethoxazole (32.7). N on fermenting gram negative strains were found resistant to ciprofloxacin (27.3), ceftazidime (9.5), cefepime (14.6), ceftriaxone (81.6), both imipenem and amikacin (18.6), trimethoprim-sulfamethoxazole (65.2), and piperacillin-tazobactam (7.5).

Conclusions. These data show a prevalent incidence of gram-positive (59.7 \%) in comparison to gram-negative (35.3\%) bacteria isolated from blood. A high percentage of methicillin-resistant staphylococci as well as ceftazidime-resistance among Enterobacteriaceae is also observed suggesting that this phenomenon requires periodically surveillance.

Received M ay 14, 2009

Accepted June 19, 2009

\section{INTRODUZIONE}

La sepsi è una causa comune di morbilità e mortalità nei pazienti critici. Le indagini microbiologiche sono, tuttavia, di solito negative e, anche se positive, sono spesso di difficile interpretazione $(5,13)$. La distinzione tra batteriemia vera e falsipositivi è importante, ma complicata da una serie di fattori relativi alla Unità di Terapia Intensiva. Le emocolture con falsi-positivi sono costose perchè richiedono spesso un maggior numero di test diagnostici, di prescrizioni di antibiotici e di prolungata degenza ospedaliera. Diversi fattori influenzano l'efficacia delle emocolture nei pazienti critici: l'uso degli antibiotici, la quantità

\section{Corresponding author: Eugenio A. Debbia}

Sezione di Microbiologia C.A. Romanzi - DISCMIT, Università di Genova

Largo Rosanna Benzi, I0 - I6I32 Genova- Italia - Tel: + 39-I0-353 38I36, 3388805256 - Fax: +39-I0-353 765I (7698)

E-mail: eugenio.debbia@unige.it; http://www.microbiologia.unige.it/dpb/debbia.htm 
di sangue prelevata, la frequenza di campionamento delle emocolture stesse e la sede di prelievo dei campioni di sangue. Inoltre, l'esito delle emocolture può essere influenzato anche dalle tecniche di preparazione della cute, dalle manipolazioni delle colture nei laboratori di microbiologia e dal tipo di sistema utilizzato per l'emocoltura. I tentativi di identificare i fattori predittivi di batteriemia vera nei pazienti critici si sono rivelati deludenti $(13,17,18)$.

Le emocolture rappresentano il test standard per diagnosticare episodi di batteriemia. I pazienti critici sono esposti a rischio più elevato di contrarre infezioni ospedaliere a causa di patologie sottostanti e della presenza di dispositivi invasivi quali il tubo endotracheale e il catetere venoso centrale (13).

L'emocoltura ha acquisito negli ultimi anni una sempre maggiore importanza, dovuta soprattutto all'aumento dell'incidenza di sepsi nei pazienti immunodepressi. Tuttavia, questo esame di laboratorio viene ancora richiesto in quantità relativamente scarsa rispetto alla reale rilevanza clinica (la sepsi è una delle più gravi sindromi infettive) e importanza diagnostica ed epidemiologica (quello che si isola dall'emocoltura è, nella grande maggioranza dei casi, il "vero" agente eziologico dell'infezione) (13). Le setticemie rappresentano, dunque, una patologia importante, con una mortalità dal 5 al $60 \%$ a seconda si tratti di sepsi o shock settico, la tempestiva e corretta terapia antibiotica migliora significativamente la prognosi dei pazienti, fino al dimezzamento del tasso di letalità $(5,15,20)$. Le principali situazioni cliniche in cui è importante eseguire un'emocoltura sono: endocarditi ed infezioni endovascolari, polmonite batterica, pielonefrite ascendente, osteomielite ematogena, meningite batterica, ascessi endoaddominali, immunodepressioni di varia origine, cateterismi venosi e arteriosi, infezioni sistemiche.

Di pari importanza all'identificazione del germe coinvolto in queste infezioni appare il fenotipo delle resistenze agli antibiotici che esso presenta. La terapia è inizialmente empirica, in attesa dei risultati del laboratorio che sono disponibili solamente dopo 24-48 ore (13). Questo comporta una buona conoscenza dei probabili patogeni coinvolti e dei loro quadri di resistenza locali che possono essere ottenuti dopo accurate indagini condotte dai laboratori di Microbiologia Clinica. Vi sono altri fattori che occorre considerare quando si ricorre all'emocoltura per la diagnosi eziologica di una batteriemia: a) nessun mezzo di coltura è valido per tutti i potenziali microrganismi e alcuni a crescita lenta (es. micobatteri) richiedono un'incubazione prolungata; b) i falsi negativi sono piuttosto frequenti nei pazienti sottoposti a terapia antibioti- ca; i campioni di sangue dovrebbero essere raccolti prima della somministrazione di antibiotici, sebbene siano disponibili dei terreni di coltura contenenti sostanze che minimizzano gli effetti di questi farmaci sulla crescita batterica; c) l'interpretazione di una singola emocoltura positiva per alcuni microrganismi (es. stafilococchi coagulasi-negativi, corinebatteri e Propionibacterium acnes) non è univoca $(3,6,8$,13-19).

Questo lavoro riporta i dati ottenuti in uno studio epidemiologico teso ad individuare i microorganismi maggiormente isolati da emocolture durante il periodo di un anno da 12 laboratorio distribuiti in modo omogeneo in un'area dell'Italia del nord, e le loro resistenze agli antibiotici.

\section{MATERIALI E METODI}

A 12 laboratori, distribuiti omogeneamente nel territorio, è stato richiesto di collezionare tutti i ceppi consecutivi isolati da campioni positivi di emocolture. I patogeni sono stati raccolti nel periodo compreso tra Febbraio 2008 e Febbraio 2009 evitando di includere nello studio ceppi già isolati da uno stesso paziente. I germi sono stati inviati successivamente ad un unico centro e preliminarmente classificati, sulla base della specie, tipo di paziente, reparto e sensibilità agli antibiotici. I microrganismi che presentavano un fenotipo di resistenze agli antibiotici multipli o a classi particolari di farmaci per una determinata specie sono stati ulteriormente purificati e saggiati nuovamente, mediante tecnica di diffusione da dischetto, come suggerito da CLSI (4) per la sensibilità agli antibiotici. La resistenza alla meticillina, in particolare, è stata confermata mediante tecnica di diffusione da dischetto utilizzando cefoxitina $(1,4)$. Se necessario è stata anche determinata la minima concentrazione inibente mediante micrometodo. Gli antibiotici per i saggi di sensibilità sono stati ottenuti da fonti commerciali Oxoid, (Milan). Sono stati collezionati 1092 microorganismi, la lista completa dei patogeni ritrovati e la loro origine è riportata nelle Tabelle 1-2 E. coli ATCC 25922, E. coli ATCC 35218, $P$. aeruginosa ATCC 27853, e S. aureus ATCC 25923, sono stati inclusi nei saggi come controlli di qualità.

\section{RISULTATI}

Sono stati isolati 1092 microrganismi, di cui 653 gram-positivi, 385 gram-negativi e 54 miceti (Tabella 1). I patogeni maggiormente rappresentati sono stati nell'ordine E. coli 234, S. epidermidis 205, S. aureus 142, S. hominis 87, Enterococcus faecalis 47, S. haemolyticus 33, Klebsiella pneumoniae 33, P. aeruginosa 32, Candida albicans 28 e Enterobacter cloacae 21. I campioni prove- 
nivano principalmente da reparti di medicina (255 isolati), rianimazione (154), chirurgia (99), malattie infettive (93), pediatria (62) e nefrologia (62) (Tabella 2).

La Tabella 3 riassume i valori delle percentuali di resistenza dei principali gruppi di ceppi isolati alle vari classi dei più importanti antibiotici saggiati. Tra gli stafilococchi la resistenza (in \%) agli antibiotici è stata rispettivamente 65.7 per oxacillina, 33.5 per gentamicina, 61.8 per azitromicina, 59.6 per eritromicina, 14.8 per cloramfenicolo, 2.0 per teicoplanina, 24.1 per trimetoprim-sulfametossazolo mentre nessun stafilococco è risultato resistente a vancomicina. La resistenza a ciprofloxacina si è attestata sul 45.2. Gli stafilococchi oxacillino-sensibili sono risultati resistenti alla penicillina nel $71.8 \%$ dei casi. e nel 10.6 per gentamicina, 6.1 per ciprofloxacina, 1.1 per teicoplanina, 38.3 per azitromicina, 31.2 per eritromicina, 0 per vancomicina e 6.1 per cloramfenicolo.

L'insensibilità alla vancomicina negli enterococchi ha raggiunto il $10.8 \%$ degli isolati. Tra questi microrganismi inoltre, la resistenza è stata 34.4 per ampicillina, 42.9 per gentamicina, 7.6 per tei- coplanina, 42.2 per ciprofloxacina, 54.7 per eritromicina e 17.5 per cloramfenicolo.

Tra le Enterobacteriaceae sono state registrate resistenze pari a 27.0 per ciprofloxacina, 74.1 per ampicillina, 15.8 per ceftazidime, 14.7 per cefoxitina,13.3 per cefepime, 15.0 per ceftriaxone, 0.95 per imipenem, 0.95 per amikacina, 5.1 per piperacillina-tazobactam e 32.7 per trimetoprim-sulfametossazolo. I germi Gram negativi non fermentanti hanno mostrato resistenze pari a 27.3 per ciprofloxacina, 9.5 per ceftazidime, 14.6 per cefepime, 81.6 per ceftriaxone, 18.6 per imipenem, 18.6 per amikacina, 65.2 per trimetoprim-sulfametossazolo e 7.5 per piperacillina-tazobactam. (Tabella 3).

La Tabella 4 riporta il numero dei microrganismi, nell'ambito degli stafilococchi, che hanno manifestato fenotipi di resistenza ad una o complessivamente a più classi di antibiotici. S. epidermidis è stata la specie che più di altre ha evidenziato fenotipi di multiresistenza agli antibiotici. Più della metà dei ceppi raccolti ha infatti dimostrato una insensibilità ad oltre 3 classi di principi attivi ed il restante numero degli isolati ha espresso resi-

Tabella 1. Distribuzione dei 1092 microrganismi isolati da emocolture

\begin{tabular}{|c|c|c|c|c|c|c|c|c|}
\hline Gram negativi & $\mathrm{N}^{\circ}$ & $\%$ & Gram positivi & $\mathrm{N}^{\circ}$ & $\%$ & Miceti & $\mathrm{N}^{\circ}$ & $\%$ \\
\hline E.coli & 234 & 60.8 & S.epidermidis & 205 & 31.4 & C.albicans & 28 & 51.9 \\
\hline K.pneumoniae & 33 & 8.6 & S.aureus & 142 & 21.7 & C.parapsilosis & 10 & 18.5 \\
\hline P.aeruginosa & 32 & 8.3 & S.hominis & 87 & 13.3 & Candida p. & 5 & 9.3 \\
\hline E.cloacae & 21 & 5.4 & E.faecalis & 47 & 7.2 & C.glabrata & 4 & 7.4 \\
\hline K.oxytoca & 13 & 3.4 & S.haemolyticus & 33 & 5.1 & C.ciferrii & 4 & 7.4 \\
\hline P. mirabilis & 11 & 2.9 & S. capitis & 19 & 2.9 & & & \\
\hline S. maltophilia & 6 & 1.6 & E faecium & 18 & 2.8 & & & \\
\hline E. aerogenes & 4 & 1.0 & S. warneri & 13 & 2.0 & & & \\
\hline A.xylosoxidans & 4 & 1.0 & S. coag neg & 11 & 1.7 & & & \\
\hline \multirow[t]{9}{*}{ S.marcescens } & 4 & 1.0 & S. pneumoniae & 7 & 1.1 & & & \\
\hline & & & S. mitis & 6 & 0.9 & & & \\
\hline & & & S.viridans & 5 & 0.8 & & & \\
\hline & & & Streptococcus spp. & 5 & 0.8 & & & \\
\hline & & & S.dysagalactiae & 5 & 0.8 & & & \\
\hline & & & M icrococcus spp. & 5 & 0.8 & & & \\
\hline & & & M.luteus & 5 & 0.8 & & & \\
\hline & & & S.simulans & 4 & 0.6 & & & \\
\hline & & & S.pyogenes & 4 & 0.6 & & & \\
\hline Altri* & 23 & 6.0 & & 32 & 4.7 & & 3 & 5.5 \\
\hline Totale & 385 & 100 & & 653 & 100 & & 54 & 100 \\
\hline
\end{tabular}

Altri: M organella morgani (3), Serratia liquefaciens (2), Pseudomonas putida (2), Ralstonia pickettii (2), Salmonella typhi (2), Alcaligenes faecalis (1), Acinetobacter baumannii (1), Acinetobacter spp. (1), Bacteroides fragilis (1), Citrobacter diversus (1), Citrobacter freundii (1), Citrobacter koseri (1), Citrobacter youngae (1), Salmonella spp. (1), G ram negativo non fermentante (1), Providencia stuartii (1), Sphingomonas paucimobilis (1).

Staphylococcus auricularis (3), Staphylococcus saprophyticus (3), Streptococcus agalactiae (3), Streptococcus bovis (3), Listeria monocytogenes (2), Streptococcus intermedius (2), Bacillus spp. (2), Bifidobacterium spp. (1),Streptococcus galloIyticus (1), Streptococcus salivarius (1), Streptococcus milleri (1), Streptococcus sangiunis (1), Streptococcus anginosus (1), Streptococcus acidominimus (1), Enterococcus durans (1), Enterococcus gallinarum (1), Enterocccus casseliflavus (1), Actinomyces spp. (1), Corynebacterium jeikeium (1), Corynebacterium spp. (1), R. mucillaginosa (1).

C. famata (2), Saccharomyces spp. (1). 
stenza alla meticillina/cefoxitina (74\%5) oltre ad almeno un altro composto. S. aureus ha dimostrato globalmente una resistenza alla meticillina del $45 \%$, mentre la multiresistenza agli antibiotici è stata osservata in circa un terzo degli isolati refrattari alla meticillina/cefoxitina. S. hominis e S. haemolyticus hanno registrato livelli di resistenza alla

Tabella 2. Origine, numero e incidenza dei ceppi più frequentemente isolati

\begin{tabular}{lrlr}
\hline Reparti & $N^{\circ}$ & $\begin{array}{l}\text { Ceppi } \\
\text { maggiormente } \\
\text { rappresentati }\end{array}$ & $\%$ \\
\hline Medicina & 255 & E.coli & 27.8 \\
\hline Rianimazione & 154 & S.epidermidis & 26.6 \\
\hline Chirurgia & 99 & E.coli & 19.2 \\
\hline Mal. Infettive & 93 & E.coli & 22.6 \\
\hline Pediatria & 62 & S.hominis & 22.6 \\
\hline N efrologia & 62 & E.coli & 29.0 \\
\hline Oncologia & 51 & S.epidermidis & 27.4 \\
\hline Ter. Intensiva & 48 & S.epidermidis & 20.8 \\
\hline Ortopedia & 21 & E.coli & 23.8 \\
\hline N eurologia & 20 & S.epidermidis & 30.0 \\
\hline Gastroenterologia & 20 & E.coli & 35.0 \\
\hline Dialisi & 20 & P.aeruginosa & 20.0 \\
\hline Area critica medicina & 19 & S.aureus & 36.8 \\
\hline Geriatria & 17 & E.coli & 35.3 \\
\hline Ginecologia & 13 & E.coli & 46.1 \\
\hline N eurochirurgia & 12 & S.haemolyticus & 25.0 \\
\hline Cure intermedie & 11 & E.coli & 54.5 \\
\hline Degenza breve & 10 & E.coli & 50.0 \\
\hline Cardiologia & 10 & E.faecalis & 30.0 \\
\hline Altri & 57 & E.coli & 28.3 \\
\hline Reparto non specifcat & 38 & & \\
\hline
\end{tabular}

Reparto non specificato 38

Totale 1092

Altri reparti: pronto soccorso (8), urologia (8),

cure domiciliari (6), chirurgia intensiva (6),

laboratorio analisi (5), pneumologia (4),

riabilitazione neuromotoria (4), fisiatria (3),

medicina d'urgenza (3), dermatologia (2), emodialisi (2),

maternità (2), residenza anziani (2), cardiochirurgia (1), psichiatria (1). meticicillina paragonabili a quelli di S.epidermidis, con elevate percentuali di stipiti multiresistenti come osservato in quest' ultima specie. Tutti gli altri stafilococchi coagulasi-negativi hanno evidenziato alti livelli di resistenza alla meticillina (dal 33 al 87\% degli stipiti isolati), senza tuttavia raggiungere livelli di multiresistenza paragonabili a quelli di $S$. epidermidis. Unica eccezione a questo comportamento generale riguarda $S$. auricularis che è risultato totalmente sensibile alla meticillina e agli altri antibiotici.

Nell'ambito degli enterococchi (Tabella 5) spicca l'elevato livello di resistenza alla vancomicina (4/18 isolati) in E. faecium, mentre lo stesso carattere in $E$. faecalis è risultato presente in 1 su 45 isolati.

Considerando i ceppi gram-negativi (Tabella 6), $E$. coli che è stato il microrganismo più frequentemente isolato, ha dimostrato multiresistenza agli antibiotici in un terzo degli stipiti saggiati, ma caratteri di resistenza concomitanti verso una o più classi di farmaci sono stati notati in oltre il 75\% degli stipiti.

Gli altri gram-negativi sia appartenenti alla famiglia delle Enterobacteriaceae si facenti parti del gruppo dei non fermentanti pur essendo stati isolati in basso numero da non più di 3 decine a numeri inferiori a 6 , hanno quasi tutti mostrato un'alta quota di ceppi multiresistenti, in molti casi nella totalità degli isolati.

\section{CONCLUSIONI}

I risultati di questa indagine confermano una maggiore incidenza generale dei microorganismi gram-positivi $(59.7 \%)$ rispetto a quelli gramnegativi (35.3\%). Il quadro è in accordo con quanto riportato dalla letteratura più recente riferita ai campioni di sangue e all'eziologia di infezioni nosocomiali $(5,13,17,20)$. E. coli primeggia non solo tra i gram-negativi ma anche in numero assoluto. L'elenco degli altri microrganismi ribadisce il ruolo non solo di $S$. aureus e $P$. aeruginosa spes-

Tabella 3. Incidenza di resistenza agli antibiotici nei principali gruppi di ceppi collezionati sul totale dei saggiati

\begin{tabular}{|c|c|c|c|c|c|c|c|c|c|c|c|c|c|c|c|c|c|c|}
\hline Ceppi ( $\mathrm{N}^{\circ}$ collezionati) & Perce & tuale $\mathrm{C}$ & di ceppi & resiste & $n+i\left(N^{c}\right.$ & saggiat & & & & & & & & & & & & \\
\hline & PEN & OXA & AMP & TZP & CRO & CAZ & FOX & FEP & IMI & AMK & GEN & CIP & SXT & TEC & VAN & AZI & ERY & $\mathrm{CHL}$ \\
\hline Stafilococchi (522) & & $\begin{array}{l}65.7 \\
(507)\end{array}$ & & & & & & & & & $\begin{array}{l}33.5 \\
(507)\end{array}$ & $\begin{array}{l}45.2 \\
(513)\end{array}$ & \begin{tabular}{|l|}
24.1 \\
$(498)$
\end{tabular} & \begin{tabular}{|l|}
2.0 \\
$(498)$
\end{tabular} & $\begin{array}{l}0.0 \\
(514)\end{array}$ & \begin{tabular}{|l|}
61.8 \\
$(249)$
\end{tabular} & \begin{tabular}{|l|}
59.6 \\
$(515)$
\end{tabular} & $\begin{array}{l}14.8 \\
(271)\end{array}$ \\
\hline Stafilococchi Oxa-S (174) & $\begin{array}{l}71.8 \\
(163)\end{array}$ & & & & & & & & & & $\begin{array}{l}10.6 \\
(164)\end{array}$ & $\begin{array}{l}6.1 \\
(164)\end{array}$ & & $\begin{array}{l}1.1 \\
(170)\end{array}$ & $\begin{array}{l}0 \\
\text { (172) }\end{array}$ & $\begin{array}{l}38.3 \\
(94)\end{array}$ & $\begin{array}{l}31.2 \\
(173)\end{array}$ & $\begin{array}{l}6.1 \\
(82)\end{array}$ \\
\hline Enterococchi (68) & & & $\begin{array}{l}34.4 \\
(61)\end{array}$ & & & & & & & & $\begin{array}{l}42.9 \\
(49)\end{array}$ & $\begin{array}{l}42.2 \\
(45)\end{array}$ & & \begin{tabular}{|l|}
7.6 \\
$(66)$
\end{tabular} & $\begin{array}{l}10.8 \\
(65)\end{array}$ & & $\begin{array}{l}54.7 \\
(64)\end{array}$ & $\begin{array}{l}17.5 \\
(40)\end{array}$ \\
\hline Enterobacteriaceae (333) & & & \begin{tabular}{|l|}
74.1 \\
$(321)$
\end{tabular} & $\begin{array}{l}5.1 \\
(295)\end{array}$ & $\begin{array}{l}15.0 \\
(313)\end{array}$ & $\begin{array}{l}15.8 \\
(316)\end{array}$ & \begin{tabular}{|l|}
14.7 \\
$(285)$
\end{tabular} & \begin{tabular}{|l|}
13.3 \\
$(316)$
\end{tabular} & $\begin{array}{l}0.95 \\
(316)\end{array}$ & $\begin{array}{l}0.95 \\
(316)\end{array}$ & & $\begin{array}{l}27.0 \\
(318)\end{array}$ & \begin{tabular}{|l|}
32.7 \\
$(324)$
\end{tabular} & & & & & $\begin{array}{l}10.4 \\
(135)\end{array}$ \\
\hline $\begin{array}{l}\text { Gram negativi non } \\
\text { fermentanti (52) }\end{array}$ & & & & $\begin{array}{l}7.5 \\
(40)\end{array}$ & $\begin{array}{l}81.6 \\
(38)\end{array}$ & $\begin{array}{l}9.5 \\
(42)\end{array}$ & $\begin{array}{l}93.3 \\
(15)\end{array}$ & $\begin{array}{l}14.6 \\
(41)\end{array}$ & $\begin{array}{l}18.6 \\
(43)\end{array}$ & $\begin{array}{l}18.6 \\
(43)\end{array}$ & & $\begin{array}{l}27.3 \\
(44)\end{array}$ & $\begin{array}{l}65.2 \\
(46)\end{array}$ & & & & & \\
\hline
\end{tabular}

Pen, penicillina; amp, ampicillina; tzp, piperacillina-tazobactam; cro, ceftriaxone; caz, ceftazidime; fox, cefoxitina; fep, cefepime; imi, imipenem; ak, amikacina; gen, gentamicina; cip, ciprofloxacina; sxt, trimethoprim-sulfametoxazolo; tec, teicoplanina; van, vancomicina, azi azitromicina; ery, eritromicina/claritromicina; chl, cloramfenicolo 
Tabella 4. Distribuzione della resistenza alla cefoxitina (FOX) e delle classi di resistenza simultanea agli antibiotici negli stafilococchi

\begin{tabular}{|c|c|c|c|c|c|c|}
\hline Specie & N . isolati & $\begin{array}{c}F O X-R \\
n\end{array}$ & $\begin{array}{c}\text { tot } \\
\%\end{array}$ & $\begin{array}{c}\text { FOX }-R \\
\mathrm{e} / 0+1 \text { classe }\end{array}$ & $\begin{array}{l}\text { FOX-R } \\
+2 \text { classi } \\
\end{array}$ & $\begin{array}{c}\text { FOX }-R \\
+>=3 \text { classi }\end{array}$ \\
\hline S. epidermidis & 205 & 152 & 74 & 24 & 44 & 84 \\
\hline S. aureus & 142 & 64 & 45 & 13 & 30 & 21 \\
\hline S. hominis & 87 & 58 & 67 & 5 & 14 & 39 \\
\hline S. haemolyticus & 33 & 29 & 87 & 2 & 1 & 26 \\
\hline S. capitis & 19 & 12 & 63 & 10 & 1 & 1 \\
\hline S. warneri & 13 & 9 & 69 & 4 & 2 & 3 \\
\hline S. simulans & 4 & 2 & 50 & - & - & 2 \\
\hline S. saprophyticus & 3 & 1 & 33 & - & - & 1 \\
\hline S.auricularis & 3 & 0 & 0 & - & - & - \\
\hline \multirow[t]{2}{*}{ OCNS } & 11 & 6 & 60 & 1 & 3 & 2 \\
\hline & 520 & 333 & 65 & 59 & 95 & 179 \\
\hline
\end{tabular}

FOX, utilizzata al posto della meticillino; OCNS, Altri stafilococchi coagulasi negativi

Tabella 5. Incidenza di resistenza alla vancomicina nelle diverse specie di enterococco isolate

\begin{tabular}{|c|c|c|c|c|c|c|c|c|c|}
\hline Specie & N . isolati & \multicolumn{4}{|c|}{ Vancomicina } & \multicolumn{4}{|c|}{ Teicoplanina } \\
\hline & & $\mathrm{S}$ & 1 & $\mathrm{R}$ & NT & $\mathrm{S}$ & $\mathrm{I}$ & $\mathrm{R}$ & NT \\
\hline E. faecalis & 47 & 44 & & 1 & 2 & 45 & & & 2 \\
\hline E. faecium & 18 & 12 & 1 & 4 & 1 & 14 & & 4 & \\
\hline Totale & 68 & 57 & 1 & 7 & 3 & 61 & & 5 & 2 \\
\hline
\end{tabular}

Tabella 6. Distribuzione delle classi di resistenza concomitante agli antibiotici nei gram-negativi

\begin{tabular}{|c|c|c|c|c|c|c|}
\hline Specie & $\mathrm{N}$. isolati & $\mathrm{S}$ & $1 \mathrm{R}$ & $2 R$ & $\geq 3 \mathrm{R}$ & NT \\
\hline E.coli & 234 & 70 & 32 & 54 & 76 & 2 \\
\hline K.pneumoniae & 33 & & 17 & 4 & 10 & 2 \\
\hline P.aeruginosa & 32 & & 5 & 7 & 20 & \\
\hline E.cloacae & 21 & 1 & & 1 & 19 & \\
\hline K.oxytoca & 13 & 2 & 8 & 1 & 2 & \\
\hline P.mirabilis & 11 & 3 & & & 7 & 1 \\
\hline S.maltophilia & 6 & & & & 1 & 5 \\
\hline E.aerogenes & 4 & & & 1 & 3 & \\
\hline S.marcescens & 4 & & & 2 & 2 & \\
\hline Achr.xylosoxidans & 4 & & & & 4 & \\
\hline M.morganii & 3 & & & 2 & 1 & \\
\hline S.liquefaciens & 2 & & & & 2 & \\
\hline C.koseri & 2 & & 2 & & & \\
\hline A.baumannii & 2 & & & 1 & 1 & \\
\hline P. putida & 2 & & & 1 & 1 & \\
\hline Ralstonia pickettii & 2 & & & & 2 & \\
\hline \multirow[t]{2}{*}{ Altri } & 10 & 1 & & 2 & 6 & 1 \\
\hline & 385 & 77 & 64 & 76 & 157 & 11 \\
\hline
\end{tabular}

Altri, C. freundii; C. youngae; P. stuartii; S. typhimurium S. typhi; Salmonella spp;

A. faecalis; S. paucimobilis; bastoncino gram-negativo non fermentante; B. fragilis

so coinvolti nelle infezioni nosocomiale ma anche di S. epidermidis, S. hominis e $S$ haemolyticus tra gli stafilococchi e di $K$. pneumoniae e Enterobacter spp tra i gram-negativi che prevalgono in incidenza su tutte le rimanenti specie batteriche. La conoscenza delle specie più frequentemente isolate da un determinato campione assume un notevole significato quando, congiuntamente ad indagini più mirate sulla diffusione della resistenza agli antibiotici, entra in gioco la terapia che è prevalentemente empirica $(>85 \%)(5,13,17)$.
Nei confronti dei gram-positivi l'incidenza di meticillino-resistenza tra gli stafilococchi è in lenta ma costante crescita anche in confronto a recenti indagini (12), ma rimane rassicurante l' attività dei glicopeptidi che, nonostante l'intenso uso di questi antibiotici, appare ancora del tutto intatta. Gli enterococchi confermano la loro refrattarietà nei confronti di molti antibiotici, ma anche per questi patogeni i glicopepdidi appaiono i farmaci di scelta. I meccanismi di resistenza agli antibiotici nei gram-negativi sono molto più 
variegati rispetto a quello dei gram-positivi. Tra le penicilline solo piperacillina-tazobactam appare dotata di un'utile potenza in vitro. Ma i dati ottenuti confermano la validità delle cefalosporine di terza generazione, dei carbapenemici e degli aminoglicosidi $(7,10,11)$, mentre la ciprofloxacina appare risentire dell'intenso uso di questa molecola nelle diverse infezioni sistemiche Nelle gravi infezioni nosocomiali la sensibilità del patogeno è infatti assai dipendente dal reparto in cui giace il paziente. Dove disponibili, tutte le indagini confermano che la terapia intensiva è l'ambiente dove è più difficile affrontare questa problematica (13, 20). La disponibilità di dati sull'incidenza locale di resistenza può favorire la decisione per ciò che concerne la scelta del b-lattamico risultando in pratica obbligato l'utilizzo dell'aminoglicoside. Per mantenere la loro utilità di indirizzo per il clinico i dati epidemiologici devono essere continuamente aggiornati e tale è il proposito maturato da questa esperienza tra i laboratori

Tra le problematiche maggiori dell'emocolture, sottolineate anche dai dati del Comitato di Batteriologia dell’AMCLI (6) vi sono gli elevati livelli di falsi sia positivi sia negativi. In questa indagine potrebbero essere chiamati in causa solo i falsi positivi, in particolare esiste un nutrito numero di patogeni gram-positivi che spesso le linee guida suggeriscono di monitorare e confermare prima di dare positiva un'emocoltura. In questo studio abbiamo suggerito a tutti i laboratori non solo di attenersi alle linee guida ma anche di avere un sostegno del clinico sulla veridicità del probabile patogeno coinvolto. Considerato l'alto numero di stafilococchi coagulasi negativi ritrovati in particolare S. epidermidis, S. hominis e $S$. haemolyticus nonché gli alti livelli di multi resistenza veicolati da questi stipiti viene da pensare ad una evoluzione di questi microrganismi simile a quella di $S$. aureus ma ancora non totalmente stimata $(2,9)$. Questo appare il dato più interessante e nello stesso tempo più preoccupante emerso da questo studio suggerendo l'attivazione di nuove indagini tese a stabilire se, come per $S$. aureus comunitario, esiste anche in questi microrganismi un'evoluzione indipendente da quella di cloni di origine comunitaria o nosocomiale.

\section{BIBLIOGRAFIA}

1. Andrews J, Brenwald N, Brown DFJ, et al. Evaluation of a $10 \mu \mathrm{g}$ cefoxitin disc for the detection of methicil- lin resistance in Staphylococcus aureus by BSAC methodology. J Antimicrob Chemoter 2005; 267: 599600

2. Archer GL, Climo MW. Staphylococcus epidermidis and other coagulase-negative staphylococci. In Mandell GL, Bennet JE, Dolin R. Principles and Practice of Infectious Disease 2005. Sixth Ed.p 23522360. Churchill Livingstone. Philadelphia, USA

3. Casolari C, Fabio A, Fabio U. Incidenza e frequenza nel sangue di batteri "inusuali “ od "esigenti”. Microbiologia Medica 2002.17:72-73

4. Clinical and Laboratory Standards Institute. Performance Standard for Antimicrobial Susceptibility Tests; Seventeenth Informational Supplement. CLSI document M100-S17, 2007. Wayne, PA

5. Cohen J. Non-antibiotic strategies for sepsis. Clin Microbiol Infect 2009; 15: 302-307

6. Comitato Studio per la Batteriologia (CosSBat) Traccia per la formulazione di linee guida per l'emocoltura 2002

7. Davies J. Microbes have the last word. EMBO Reports 2007; 8: 616-621

8. Farina, C. "I miceti". Microbiologia Medica 2002.17:74

9. Li M, Diep BA, Villaruz AE, et al. Evolution of virulence in epidemic community-associated methicillinresistant Staphylococcus aureus. Proc Natl Acad Sci U S A. 2009 ; 106:5883-8

10. Livermore DM. Bacterial resistance: origins, epidemiology, and impact. Clin Infect Dis 2003; 36 (Suppl 1): S11-23

11. Livermore DM., Pearson A. Antibiotic resistance: location, location, location. Clin Microbiol Infect 2007: 13 (Suppl 2): 7-16

12. Maioli E, Bandettini R, Battolla E, et al. Indagine sulla diffusione di stafilococchi resistenti alla meticillina in Liguria. Microbiologia Medica 2006; 21: 316321

13. Munford RS. Sepsis, severe sepsis, and septic shock. In Mandell GL, Bennet JE, Dolin R. Principles and Practice of Infectious Disease2005. Sixth Ed.p 906926. Churchill Livingstone. Philadelphia, USA

14. Paisley, JW, BA Lauer. Pediatric blood cultures. Clin Lab Med 1994; 14:17-30

15. Paradisi F, Corti G, Sbaragli S. Il punto di vista dell'infettivologo. Microbiologia Medica 2002.17:71-72

16. Reimer LG, Wilson ML, Weinstein MP. Update on detection of bacteremia and fungemia. Clin Microbiol Rev 1997; 10:444-465

17. Shafazand S, Weinacker AB. Emocolture in Unità di Terapia Intensiva. Miglioramento d'utilizzo e d'efficacia CHEST Edizione Italiana 2003; 4:64-74

18. Thomson RB jr. Specimen collection, transport and processing: bacteriology. In Murray PR, Baron EJ, Jorgensen J.H., Landry ML, Pfaller MA, eds. Manual of Clinical Microbiology 2007. 9th ed. P. 291-333 Washington, DC: American Society for Microbiology Press

19. Tortoli E. La ricerca colturale di micobatteri nel sangue. Microbiologia Medica 2002; 17:73

20. Vincent JL, Sakr Y, Sprung CL, et al. Sepsis in European intensive care units: results of the SOAP study. Crit Care Med 2006; 34: 344-353 